\title{
Young peoples' views of online historical archives
}

\author{
Holly Crossen-White ${ }^{1}$ and Angela Turner-Wilson ${ }^{1}$ \\ ${ }^{1}$ Bournemouth University, Bournemouth, UK \\ hcrossen@bournemouth.ac.uk \\ aturnerwilson@bournemouth.ac.uk
}

\begin{abstract}
Digitized collections are 'a rich source of instructional material for history teachers' [1 p314] but it has been noted these 'remained largely underused'. There is 'a growing interest in both improving the user experience and in justifying the creation of digital collections to multiple stakeholders' [2 p339]. Within the UK an estimated £130m has been invested in digitisation projects [3]. Recent changes to the UK National Curriculum for history have placed greater emphasis upon the importance of understanding methods of historical enquiry and the use of evidence. Therefore, the digitized collections created by this investment should be a vital classroom tool. This study set out to investigate within the UK the level of awareness of these resources and their value to young learners aged 8-16 years. There were two stages to this qualitative study. Stage one was the delivery of a workshop which introduced young learners to a range of digital archives. After this the young people were given a period of time to use the online archives and explore the difference forms of historical evidence. Stage two involved focus groups with a sample of the young people during which participants were asked to discuss their end-user experience. Key findings included enhanced personal learning experience, development of a personal connection to the past, and identification of issues related to usability and practical application in a classroom learning context.
\end{abstract}

Keywords: Digital Heritage, Learning Technology, Self-directed Learning, UK Research, Young Learners.

\section{Introduction}

Digital technology has transformed the ways in which the past can be explored [4-5]. Within this social shift historical evidence has become much more accessible [6] and has offered the opportunity to make links with this type of material in ways that previously could never 
have been attempted. One example from the UK would be the London Lives digital project which resulted in a greater understanding of the lives of individuals living in the capital between 1690 and 1800 [7]. However it is also recognised that with this ability to link different sources of historical evidence comes ethical issues for researchers as it is now much easier to 'discover' the personal stories of long forgotten individuals [8]. It is this potential of online archives to bring the past back to life that makes them a powerful learning resource.

To discover, anecdotally, that some teachers were unaware of these resources and young learners were potentially not accessing them was an interesting insight. This was particularly relevant given that between the late 1990s and 2005 an estimated $£ 130$ million had been spent within the UK on creating these types of resources [3]. It would appear that this investment was being underutilised by the education sector.

A search of the literature indicated that studies largely focused upon who were using specific collections with the purpose of capturing data that would lead to technical refinements [2]. Other studies indicated that there was high usage by academics within higher education [9]. However little research had been undertaken into the personal experience of other end-user groups, or indeed who they were, their purpose of accessing and satisfaction with results.

\section{Study Design}

A two stage study was undertaken that involved the delivery of a workshop session to young learners in stage one. This was followed by a period when the young people could access and use the online archives by themselves. The participants were asked to keep a record of the online archives they visited, what they searched within this, what content they found and note how helpful or informative the search had been. These personal records were then used to construct a sample of young people who were invited to take part in the focus groups which were stage two of the study. The focus groups were audio recorded and transcribed and thematic analysis undertaken. A key limitation of the study was the small sample size which made the findings not generalizable, however the intension of the study was to scope out topics for further research, and it should be noted that the findings offer some valuable insights. 


\section{Sample}

Participants for the study were aged between 8 and 16 years and drawn from two sources, one a youth group and the other a primary school. Some of the participants were not fully engaged with mainstream education and some among the sample reported no interest in history prior to joining the study. In total 42 young people participated in stage one and 20 in stage two. Ten of the young people from the youth group who were aged between 12 and 16 years took part in a focus group, and 10 from the primary school. These participants were all aged between 8 and 9 years. The sample was randomly selected based upon their recorded usage of the online archives with three being drawn from each of three groups: high, medium or low usage. A tenth was selected at random from the remaining personal usage records.

\section{Findings}

- None of the young people were aware of digital archives so the workshop session was their first introduction to this type of learning resource.

- Although a number of the participants self-reported that history held no real interest all of the participates used the archives after their workshop session and several talked of how using the online archives had captured their interest which led them to search for particular historical content.

- The young people enjoyed the opportunities that the online archive offered for self-directed learning.

- The young people were able to recall in detail the content of the historical evidence that they viewed, even though a period of time had elapsed between access and the focus group.

- The participants valued the online archives and wanted to use them further in their learning but recognised how it might be difficult to regularly use during lesson time due to practical issues such as access to computers. However, all age groups identified ways these resources could be incorporated into their learning experience.

- The participants discussed usability issues and indicated what features were of value.

- There appeared to be a raised self-esteem among the learners. Many discussed how pleased they were that access to the online 
archives had given them the opportunity to find historical evidence to use in their topic of study that others in their group did not know.

\section{Conclusion}

Online historical archives are a valuable resource to young learners. The content on offer has the potential to nurture students' enthusiasm and to engage them in self-direct learning. In addition it can raise their self-esteem by enabling them to discover and share their new knowledge with their peers and others. Given the positive feedback from young learner about these resources future research is required to identify how teachers can be made more aware of and practically use these resources within the classroom environment.

\section{References}

1. Pattuelli, M.C.: Modeling a Domain Ontology for Cultural Heritage Resources: A User-Centred Approach. Journal of the American Society for Information Science and Technology 62 (2), 314-342 (2011).

2. Kelly, E.J.: Assessment of Digitized Library and Archives Materials: A Literature Review. Journal of Web Librarianship 8 (4), 384-403 (2014).

3. JISC Digitisation in the UK: the case for a UK framework, JISC (2005).

4. Floud, R. Shoemaker, R.,Spaeth, D.: From computers and history to digital history: a retrospective. http://www.history.ac.uk/podcasts/digital-history/computersand-history-digital-history-retrospective (2013).

5. Allen, R.B. Sieczkiewicz, R.: How historians use historical newspapers. In: Proceedings of the American Society for Information Science and Technology Pittsburgh, USA, October 2010, 47, 1-4. Wiley 2010. 
6. Tanner, S.: The value and impact of digitized resources for learning, teaching, research and enjoyment. In: Hughes LM (eds) Evaluating and measuring the value, use and impact of digital collections. Facet, London, 103-120 (2012).

7. Hitchcock, T. Shoemaker, R. Howard, S. McLaughlin, J. et al.,: London Lives, 1690-1800. www.londonlives.org (2012).

8. Crossen-White, H.: Using digital archives in historical research: What are the ethical concerns for a 'forgotten' individual? Research Ethics. 11 (2), 108-119 (2015).

9. Alcencer-Brayner, A.: British Library Digital Scholarship survey: how are users engaging with our digital content? http://blogs.bl.uk/digital-scholarship/2014/11/bl-digitalscholarship-survey-how-are-users-engaging-with-our-digitalcontent.html last accessed 2017/03/21 